\title{
Microsatellite instability-high is rare events in refractory pediatric solid tumors
}

\author{
Taro Yoshida ${ }^{1}$, Hideki Muramatsu ${ }^{1}$, Manabu Wakamatsu ${ }^{1}$, Rieko Taniguchi ${ }^{1}$, Daisuke \\ Ichikawa $^{1}$, Masato Nakaguro ${ }^{1}$, Atsushi Natsume ${ }^{1}$, and Yoshiyuki Takahashi ${ }^{1}$ \\ ${ }^{1}$ Nagoya University Graduate School of Medicine Faculty of Medicine
}

July 20, 2020

Microsatellites are a set of repeating base sequences of one to several bases in a chromosome. In general, mismatch repair (MMR) proteins correct the base mismatches that occur during DNA replication. However, tumor cells with deficient MMR function accumulate genetic mutations and cause changes in the repeat counts in microsatellite sites, and such a status is referred to as microsatellite instability (MSI)-high status. According to recent research, MSI-high status is associated with responsiveness to therapies with immune checkpoint inhibitors [1].

MSI status has been well described in various adult solid tumors; for instance, one of the largest studies has demonstrated that 1188 (9.9\%) of 12,019 patients exhibited an MSI-high signature in various types of tumors [2]. However, there are no sufficient investigations on the MSI status in pediatric solid tumors, except those on limited tumor subtypes, including glioblastoma and medulloblastoma [3, 4]. Herein, we investigated the MSI status in pediatric patients with various solid tumors who died due to the tumor and also evaluated the potential of immune checkpoint inhibitors in refractory pediatric solid tumors.

From April 2000 to May 2019, a total of 334 pediatric patients with solid tumors were admitted to the Nagoya University Hospital (Table 1 ). Although the majority of patients survived, 74 (22\%) died, including 68 due to relapse or refractory tumor, 4 due to pulmonary complications after stem cell transplantations, and 2 due to infection after chemotherapies. We retrospectively analyzed the formalin-fixed paraffin-embedded tumor tissues of $40(54 \%)$ of the 74 patients who died to assess the MSI status (Supplemental Table 1, Supplemental Figure 1 ) using five multiplexed markers for determining the MSI-high phenotype (BAT25, BAT-26, MONO-27, NR-21, and NR-24) (Supplemental methods). Results demonstrated that 36 cases were microsatellite-stable and none of the patients had an MSI-high status; however, this observation could not be confirmed for the remaining four patients because of poor sample quality.

These results indicate that MSI-high status is rare in pediatric patients with solid tumors who die of the disease. Therefore, surveillance of MSI status in children with refractory/relapsed solid tumors might have a limited role in predicting the responsiveness to immune checkpoint inhibitors.

\section{References}

1. Yamashita $\mathrm{H}$, et al. Microsatellite instability is a biomarker for immune checkpoint inhibitors in endometrial cancer. Oncotarget. 2018; 9(5): 5652-5664.

2. Le, D.T, et al. Mismatch repair deficiency predicts response of solid tumors to PD-1 blockade. Science. 2017; 357(6349): 409-413.

3. Viana-Pereira M, et al. Analysis of microsatellite instability in medulloblastoma. Neuro Oncol. 2009; 11(5): 458-67. 
4. Viana-Pereira M, et al. Microsatellite instability in pediatric high grade glioma is associated with genomic profile and differential target gene inactivation. PLoS One. 2011; 6(5): e20588.

\section{Hosted file}

Table1 final..docx available at https://authorea.com/users/344206/articles/470860microsatellite-instability-high-is-rare-events-in-refractory-pediatric-solid-tumors 